\title{
Convexity analysis of active contour problems
}

\author{
Christos Davatzikos ${ }^{\mathrm{a}, *}$, Jerry L. Prince ${ }^{\mathrm{a}, \mathrm{b}}$ \\ ${ }^{a}$ Department of Radiology and Radiological Science, Johns Hopkins University School of Medicine, Baltimore, MD 21287, USA \\ ${ }^{\mathrm{b}}$ Department of Electrical and Computer Engineering, Johns Hopkins University, Baltimore, MD 21218, USA
}

Received 5 March 1997; received in revised form 4 January 1998; accepted 22 January 1998

\begin{abstract}
A general active contour formulation is considered and a convexity analysis of its energy function is presented. Conditions under which this formulation has a unique solution are derived; these conditions involve both the active contour energy potential and the regularization parameters. This analysis is then applied to four particular active contour formulations, revealing important characteristics about their convexity, and suggesting that external potentials involving center-of-mass computations may be better behaved than the usual potentials based on image gradients. Our analysis also provides an explanation for the poor convergence behavior at concave boundaries and suggests an alternate algorithm for approaching these types of boundaries. (C) 1999 Elsevier Science B.V. All rights reserved.
\end{abstract}

Keywords: Convexity analysis; Active contour problems; Energy function; Deformable models

\section{Introduction}

Active contours were originally described [1] as a physically-based model for finding boundaries and tracking their motion, and were explored more recently in a curve evolution framework [2-4]. They have been successfully used in a wide variety of applications [5-15]. The main advantage of active contours is that they are topologically isomorphic to the features they seek, namely object boundaries. As a result, they require no edge linking $[16,17]$, and they are robust to low contrast, noise, and gaps or spurious branches in boundaries. A main disadvantage is that their convexity properties are poorly understood. Specifically, it has been noted in the past that active contour models are nonconvex, and that solutions are often local rather than global minima $[1,7,13,18-20]$. This accounts for the phenomenon of convergence to the wrong result.

Further, the local solutions often involve discontinuities or "splits" in the final contour [13]. Our main goal in this paper is to understand the nature of the convergence problem through a study of the convexity of the active contour energy function. In particular, we consider the following question: under what conditions is the active contour energy function convex? We start by stating a fairly general active contour formulation in the continuum. We then discretize this formulation and derive a convexity condition for the discrete model. This condition involves the

\footnotetext{
* Corresponding author.
}

regularization parameters of the problem-these prescribe the elasticity and rigidity of the active contour-and local object characteristics such as curvature. The fact that the object itself partly determines the convexity of the formulation is unavoidable, and is partly what accounts for the often-observed excellent convergence in some regions and very bad convergence in other regions.

To demonstrate the utility of our convexity analysis, we apply it to four active contour formulations differing only in their definition of external energy. Since the primary application of our research is to cortical brain mapping [13], our four models focus on the detection of ribbons (which are good models for the cortex in two-dimensional magnetic resonance images of the brain). This framework includes the usual boundary mapping problem as a special case since the ribbons can be made very thin. Therefore, boundaries resulting from convolutional or morphological edge detection operations [21,22] can also be studied within this framework. The four active contour formulations include two that are modifications of the potentials commonly used in the "snake" models [1,23] and two that were introduced in Refs. [13,20,24]. This analysis reveals the existence of subdomains of convexity and suggests alternative active contour algorithms, which seek solutions within a subdomain of convexity, thereby avoiding local minima.

The organization of the paper is as follows. Section 2 describes a general discrete active contour variational formulation, and Section 3 develops a convexity analysis for this formulation. Section 4 applies the convexity 
analysis of Section 3 to four potentials for parameterizing ribbons. Finally Section 5 summarizes the key results of the paper.

\section{Active contour model}

An active contour is a curve $\mathbf{x}(s)=(x(s), y(s)), s \in[0,1]$, that moves through image data to minimize an energy [1]

$\varepsilon=\varepsilon_{\mathrm{I}}+\varepsilon_{\mathrm{E}}$,

where $\varepsilon_{\mathrm{I}}$ is the internal energy and $\varepsilon_{\mathrm{E}}$ is the external energy, defined as

$\varepsilon_{\mathrm{I}}=K_{0} \int_{0}^{1}\left\|\mathbf{x}^{\prime}(s)\right\|^{2} \mathrm{~d} s+K_{1} \int_{0}^{1}\left\|\mathbf{x}^{\prime \prime}(s)\right\|^{2} \mathrm{~d} s$,

$\varepsilon_{\mathrm{E}}=\int_{0}^{1} P(\mathbf{x}(s)) d s$

Here, the primes denote derivatives with respect to $s$. The function $P(\mathbf{x})=P(x, y)$, called the potential, is derived from the image so that it has a minimum value along the boundaries of interest. It is assumed in this paper to be twice differentiable. We will refer to the variational problem above as VP.

It can be shown using calculus of variations that a curve minimizing $\varepsilon$ must satisfy the Euler equations [25]

$-\nabla_{\mathbf{x}} P(\mathbf{x}(s))+2 K_{0} \mathbf{x}^{\prime \prime}(s)-2 K_{1} \mathbf{x}^{\prime \prime \prime \prime}(s)=0$.

This equation represents a force balance condition, whereby the negative gradient of the potential balances the sum of two internal forces arising from the elasticity and rigidity of the active contour. To fully specify the model, we assume the following boundary conditions

$\mathbf{x}(0)=\mathbf{l}, \mathbf{x}(1)=\mathbf{r}$

$\mathbf{x}^{\prime}(0)=\mathbf{l}_{p}, \mathbf{x}^{\prime}(1)=\mathbf{r}_{p}$.

In this paper, we consider a discrete active contour model defined using finite difference approximations of the derivatives in Eqs. (2a) and (2b). Let the active contour be modeled as a collection of points $\mathbf{x}_{i}, i=0, \ldots, N$, where $\mathbf{x}_{i}=\mathbf{x}(i / N)$. The free values of the discrete curve, i.e. those not fixed by the boundary conditions, are represented by the vector

$\mathbf{d}=\left[\mathbf{x}_{1}^{\mathrm{T}}, \ldots, \mathbf{x}_{N-1}^{\mathrm{T}}\right]^{\mathrm{T}}$.

Then we obtain the discrete approximation of the energy function $\varepsilon$,

$\tilde{\varepsilon}(\mathbf{d})=\tilde{\varepsilon}_{\mathrm{I}}(\mathbf{d})+\tilde{\varepsilon}_{\mathrm{E}}(\mathbf{d})$ where

$$
\begin{aligned}
\tilde{\varepsilon}_{\mathrm{I}}(\mathbf{d})= & K_{0} N \sum_{i=1}^{N-2}\left\|\mathbf{x}_{i+1}-\mathbf{x}_{i}\right\|^{2}+K_{0} N \\
& \times\left(\left\|\mathbf{x}_{1}-\mathbf{l}\right\|^{2}+\left\|\mathbf{x}_{N-1}-\mathbf{r}\right\|^{2}\right) \\
& +K_{1} N^{3}\left(\sum_{i=2}^{N-2}\left\|\mathbf{x}_{i+1}+\mathbf{x}_{i-1}-2 \mathbf{x}_{i}\right\|^{2}+\left\|\mathbf{x}_{2}+\mathbf{l}-2 \mathbf{x}_{1}\right\|^{2}\right. \\
& \left.+\mathbf{x}_{N-2}+\mathbf{r}-2 \mathbf{x}_{N-1} \|^{2}\right)+\frac{1}{2} K_{1} N^{3} \\
& \times\left(\left\|\mathbf{x}_{1}+\mathbf{x}_{-1}-2 \mathbf{l}\right\|^{2}+\left\|\mathbf{x}_{N-1}+\mathbf{x}_{N+1}-2 \mathbf{r}\right\|^{2}\right),
\end{aligned}
$$

$\tilde{\varepsilon}_{\mathrm{E}}(\mathbf{d})=\frac{1}{N} \sum_{i=1}^{N-1} P\left(\mathbf{x}_{i}\right)+\frac{1}{2 N}[P(\mathbf{l})+P(\mathbf{r})]$,

and where

$\mathbf{x}_{-1}=\mathbf{l}-\frac{1}{N} \mathbf{l}_{p}$,

$\mathbf{x}_{N+1}=\mathbf{r}+\frac{1}{N} \mathbf{r}_{p}$

are constant vectors determined from the boundary conditions.

The necessary condition for $\mathbf{d}$ to be a minimizer of $\tilde{\varepsilon}(\mathbf{d})$, i.e. the discrete Euler equation, is

$$
\begin{aligned}
\nabla \varepsilon(\mathbf{d})= & 2 K_{0} N \mathbf{A}_{1} \mathbf{d}-K_{0} N \mathbf{b}_{1}+2 K_{1} N^{3} \mathbf{A}_{2} \mathbf{d}-K_{1} N^{3} \mathbf{b}_{2} \\
& +\mathbf{p}=0 .
\end{aligned}
$$

Here,

$\mathbf{b}_{1}=\left[2 \mathbf{l}^{\mathrm{T}}, \boldsymbol{0}^{\mathrm{T}}, \ldots, \boldsymbol{0}^{\mathrm{T}}, 2 \mathbf{r}^{\mathrm{T}}\right]^{\mathrm{T}}$

$\mathbf{b}_{2}=\left[5 \mathbf{l}^{\mathrm{T}}+\frac{1}{N} \mathbf{l}_{p}^{\mathrm{T}},-2 \mathbf{l}^{\mathrm{T}}, \boldsymbol{0}^{\mathrm{T}}, \ldots, \boldsymbol{0}^{\mathrm{T}},-2 \mathbf{r}^{\mathrm{T}}, 5 \mathbf{r}^{\mathrm{T}}-\frac{1}{N} \mathbf{r}_{p}^{\mathrm{T}}\right]^{\mathrm{T}}$

$\mathbf{p}=\left[\nabla P^{\mathrm{T}}\left(\mathbf{x}_{1}\right), \ldots, \nabla P^{\mathrm{T}}\left(\mathbf{x}_{N-1}\right)\right]^{\mathrm{T}}$

and where

$\mathbf{A}_{1}=\left[\begin{array}{ccc}\mathbf{B}_{1} & \vdots & 0 \\ \cdots & \vdots & \cdots \\ 0 & \vdots & \mathbf{B}_{1}\end{array}\right]$,

and

$\mathbf{A}_{2}=\left[\begin{array}{ccc}\mathbf{B}_{2} & \vdots & 0 \\ \cdots & \vdots & \cdots \\ 0 & \vdots & \mathbf{B}_{2}\end{array}\right]$,

where $\mathbf{B}_{1}$ is a symmetric Toeplitz tridiagonal matrix whose 
first row is $[2,-1, \ldots, 0]$ and

$$
\mathbf{B}_{2}=\left[\begin{array}{cccccc}
5.5 & -4 & 1 & 0 & \cdots & 0 \\
-4 & 6 & -4 & 1 & \ddots & \vdots \\
1 & -4 & 6 & -4 & \ddots & 0 \\
0 & 1 & -4 & 6 & \ddots & 1 \\
\vdots & \ddots & \ddots & \ddots & \ddots & -4 \\
0 & \cdots & 0 & 1 & -4 & 5.5
\end{array}\right] .
$$

If $\tilde{\varepsilon}(\mathbf{d})$ is strictly convex then Eq. (9) is also a sufficient condition, and $\mathbf{d}$ is the unique minimizer of $\tilde{\varepsilon}(\mathbf{d})$.

In what follows, the domain where the potential $P$ is defined is denoted by $\mathcal{R}$. The domain $\mathcal{D}$ in which $\mathbf{d}$ is defined is then given by

$$
\begin{aligned}
\mathcal{D}= & \left\{\mathbf{e}=\left[\mathbf{e}_{1}^{\mathrm{T}}, \ldots, \mathbf{e}_{N-1}^{\mathrm{T}}\right]^{\mathrm{T}} \in \mathbb{R}^{2 N-2} \mid \mathbf{e}_{i} \in \mathcal{R},\right. \\
& i=1,2, \ldots, N-1\} .
\end{aligned}
$$

The domains $\mathcal{R}$ and $\mathcal{D}$ depend on the potential $P$, which in turn is derived from the image data.

\section{Convexity analysis}

The question we address in this section is this: when is $\tilde{\varepsilon}(\mathbf{d})$ strictly convex? This question is equivalent to the question: when is a solution satisfying Eq. (9) the unique minimizer of $\tilde{\varepsilon}(\mathbf{d})$ ? Practically, if we know that $\tilde{\varepsilon}(\mathbf{d})$ is convex then any solution satisfying Eq. (9) is also the globally optimal solution - it is not a local minimum.

Our goal in this section is to find a sufficient condition for the strict convexity of $\tilde{\varepsilon}(\mathbf{d})$. First, we note that if the Hessian matrix $\mathbf{H}$ of $\tilde{\varepsilon}(\mathbf{d})$ is positive definite at each point in $\mathcal{D}$ then $\tilde{\varepsilon}(\mathbf{d})$ is strictly convex. Since $\mathbf{H}$ is guaranteed to be positive definite if the eigenvalues of $\mathbf{H}$ are strictly positive, we have that $\tilde{\varepsilon}(\mathbf{d})$ is strictly convex if the smallest eigenvalue of $\mathbf{H}$ is greater than zero, i.e.

$\lambda_{\min }(\mathbf{H})>0$.

Now letting $\mathbf{H}_{\mathrm{E}}$ and $\mathbf{H}_{\mathrm{I}}$ denote the Hessian matrices of $\tilde{\varepsilon}_{\mathrm{E}}(\mathbf{d})$ and $\tilde{\varepsilon}_{\mathrm{I}}(\mathbf{d})$, respectively, we have from Eq. (6) that

$\mathbf{H}=\mathbf{H}_{\mathrm{I}}+\mathbf{H}_{\mathrm{E}}$.

Using the fact that the smallest eigenvalue of the sum of two symmetric matrices is greater than or equal to the sum of the smallest eigenvalues of the two matrices, we have the following sufficient condition: $\widetilde{\varepsilon}(\mathbf{d})$ is strictly convex if

$\lambda_{\min }\left(\mathbf{H}_{\mathrm{I}}\right)+\lambda_{\min }\left(\mathbf{H}_{\mathrm{E}}\right)>0$.

In the following sections we find expressions for the minimum eigenvalues of $\mathbf{H}_{\mathrm{E}}$ and $\mathbf{H}_{\mathrm{I}}$

\subsection{Minimum eigenvalue of $\mathbf{H}_{\mathrm{I}}$}

From Eq. (7a) and after some algebra we find that the eigenvalues of $\mathbf{H}_{\mathrm{I}}$ coincide with those of the matrix $2 K_{0} N \mathbf{A}_{1}$ $+2 K_{1} N^{3} \mathbf{A}_{2}$ (see also Eq. (9)). From Eqs. (10) and (11) we see that the eigenvalues of $\mathbf{A}_{1}$ coincide with those of $\mathbf{B}_{1}$ and those of $\mathbf{A}_{2}$ with those of $\mathbf{B}_{2}$; therefore, we conclude that

$\lambda_{\min }\left(\mathbf{H}_{\mathrm{I}}\right) \geq 2 K_{0} N \lambda_{\min }\left(\mathbf{B}_{1}\right)+2 K_{1} N^{3} \lambda_{\min }\left(\mathbf{B}_{2}\right)$

The eigenvalues of $\mathbf{B}_{1}$ can be found through a recursion formula [26], giving the following minimum eigenvalue

$\lambda_{\min }\left(\mathbf{B}_{1}\right)=2(1-\cos (\pi / N))$.

The eigenvalues of $\mathbf{B}_{2}$ satisfy a double recursion formula which does not have an explicit solution. A lower bound can be readily found, however, as follows. It is straightforward to show that

$\mathbf{B}_{2}=\mathbf{B}_{1}^{2}+\frac{1}{2} \mathbf{z}_{1} \mathbf{z}_{1}^{\mathrm{T}}+\frac{1}{2} \mathbf{z}_{2} \mathbf{z}_{2}^{\mathrm{T}}$,

where $\mathbf{z}_{1}=[1,0, \ldots, 0]^{\mathrm{T}}$ and $\mathbf{z}_{2}=[0, \ldots, 0,1]^{\mathrm{T}}$. Since $\lambda_{\min }\left(\mathbf{z}_{1}\right.$ $\left.\mathbf{z}_{1}^{\mathrm{T}}\right)=\lambda_{\min }\left(\mathbf{z}_{2} \mathbf{z}_{2}^{\mathrm{T}}\right)=0$, the minimum eigenvalue of $\mathbf{B}_{2}$ must satisfy $\lambda_{\min }\left(\mathbf{B}_{2}\right) \geq \lambda_{\min }\left(\mathbf{B}_{1}^{2}\right)$. Since $\mathbf{B}_{1}$ is positive definite, we have $\lambda_{\min }\left(\mathbf{B}_{1}^{2}\right)=\left(\lambda_{\min }\left(\mathbf{B}_{1}\right)\right)^{2}$ which yields

$\lambda_{\min }\left(\mathbf{B}_{2}\right) \geq\left(\lambda_{\min }\left(\mathbf{B}_{1}\right)\right)^{2}$.

Substituting Eqs. (15) and (16) into Eq. (14) gives the final result

$\lambda_{\min }\left(\mathbf{H}_{\mathrm{I}}\right) \geq 4 K_{0} N(1-\cos (\pi / N))+8 K_{1} N^{3}(1-\cos (\pi / N))^{2}$.

\subsection{Minimum eigenvalue of $\mathbf{H}_{\mathrm{E}}$}

Taking second partial derivatives of $\tilde{\varepsilon}_{\mathrm{E}}(\mathbf{d})$ and using the fact that

$\nabla_{\mathbf{x}_{j}} P\left(\mathbf{x}_{i}\right)=0, i \neq j$,

it is not hard to see that $\mathbf{H}_{\mathrm{E}}$ is a block diagonal matrix with diagonal entries

$D_{i}=\frac{1}{N}\left[\begin{array}{cc}P_{x x}\left(\mathbf{x}_{i}\right) & P_{x y}\left(\mathbf{x}_{i}\right) \\ P_{x y}\left(\mathbf{x}_{i}\right) & P_{y y}\left(\mathbf{x}_{i}\right)\end{array}\right] i=1, \ldots, N-1$.

Thus, the eigenvalues of $\mathbf{H}_{\mathrm{E}}$ can be determined by finding the eigenvalues of these $2 \times 2$ matrices for $i=1, \ldots N-1$. A direct solution yields

$$
\begin{aligned}
\lambda_{i 1}= & \frac{1}{2 N}\left(P_{x x}\left(\mathbf{x}_{i}\right)+P_{y y}\left(\mathbf{x}_{i}\right)\right. \\
& \left.+\sqrt{\left(P_{x x}\left(\mathbf{x}_{i}\right)-P_{y y}\left(\mathbf{x}_{i}\right)\right)^{2}+4 P_{x y}^{2}\left(\mathbf{x}_{i}\right)}\right),
\end{aligned}
$$

$\lambda_{i 2}=\frac{1}{2 N}\left(P_{x x}\left(\mathbf{x}_{i}\right)+P_{y y}\left(\mathbf{x}_{i}\right)\right.$

$$
\left.-\sqrt{\left(P_{x x}\left(\mathbf{x}_{i}\right)-P_{y y}\left(\mathbf{x}_{i}\right)\right)^{2}+4 P_{x y}^{2}\left(\mathbf{x}_{i}\right)}\right),
$$


for $i=1, \ldots, N-1$. The minimum eigenvalue is

$\lambda_{\min }\left(\mathbf{H}_{\mathrm{E}}\right)=\min \left\{\lambda_{i 1}, \lambda_{i 2}, i=1, \ldots, N-1\right\}$.

\subsection{Convexity condition}

Using Eqs. (17) and (19) in Eq. (13) gives the following general convexity condition: $\tilde{\varepsilon}(\mathbf{d})$ is strictly convex on $\mathcal{D}$ if

$$
\begin{aligned}
& \min _{1 \leq i \leq N-1}\left\{\lambda_{i 1}, \lambda_{i 2}\right\}+4 K_{0} N(1-\cos (\pi / N)) \\
& +8 K_{1} N^{3}(1-\cos (\pi / N))^{2}>0
\end{aligned}
$$

is satisfied for all $\mathbf{d}$ in $\mathcal{D}$. By multiplying both sides of Eq. (20) by $N / 2$ we obtain

$$
\begin{aligned}
& \frac{1}{2} \min _{1 \leq i \leq N-1}\left\{N \lambda_{i 1}, N \lambda_{i 2}\right\}+2 K_{0} N^{2}(1-\cos (\pi / N)) \\
& +4 K_{1} N^{4}(1-\cos (\pi / N))^{2}>0 .
\end{aligned}
$$

From Eq. (18a) and Eq. (18b), we see that the terms $N \lambda_{i 1}$ and $N \lambda_{i 2}$ are independent of $N$. We can go a step further to eliminate the dependence of this expression on the specific location of the points $\mathbf{x}_{i}, i=1, \ldots, N-1$. To do this we define

$$
\begin{aligned}
h_{1}(\mathbf{x})= & \frac{1}{4}\left(P_{x x}(\mathbf{x})+P_{y y}(\mathbf{x})\right. \\
& \left.+\sqrt{\left(P_{x x}(\mathbf{x})-P_{y y}(\mathbf{x})\right)^{2}+4\left(P_{x y}(\mathbf{x})^{2}\right.}\right), \\
h_{2}(\mathbf{x})= & \frac{1}{4}\left(P_{x x}(\mathbf{x})+P_{y y}(\mathbf{x})\right. \\
& \left.-\sqrt{\left(P_{x x}(\mathbf{x})-P_{y y}(\mathbf{x})\right)^{2}+4\left(P_{x y}(\mathbf{x})^{2}\right.}\right),
\end{aligned}
$$

which leads directly to our final convexity condition: the energy function of VP is strictly convex for every active contour in a subset $\mathcal{R}^{\prime} \subseteq \mathcal{R}$ if

$$
\begin{aligned}
& A\left(\mathcal{R}^{\prime}\right)+2 K_{0} N^{2}(1-\cos (\pi / N)) \\
& \quad+4 K_{1} N^{4}(1-\cos (\pi / N))^{2}>0,
\end{aligned}
$$

where

$A\left(\mathcal{R}^{\prime}\right)=\min _{\mathbf{x} \in \mathcal{R}^{\prime}}\left\{h_{1}(\mathbf{x}), h_{2}(\mathbf{x})\right\}$.

The use of a subset $\mathcal{R}^{\prime}$ of $\mathcal{R}$ allows for the possibility that we may only be interested in convexity on a smaller set than the entire domain of definition of the potential. The remaining issue is how to determine $A\left(\mathcal{R}^{\prime}\right)$.

We note that similar results, for specific potentials, however, were described in Refs. [13,27].

\subsection{Approximations}

From Eqs. (22a), (22b), (23) and (24), it is clear that convexity of $\tilde{\varepsilon}(\mathbf{d})$ depends on the regularization coefficients

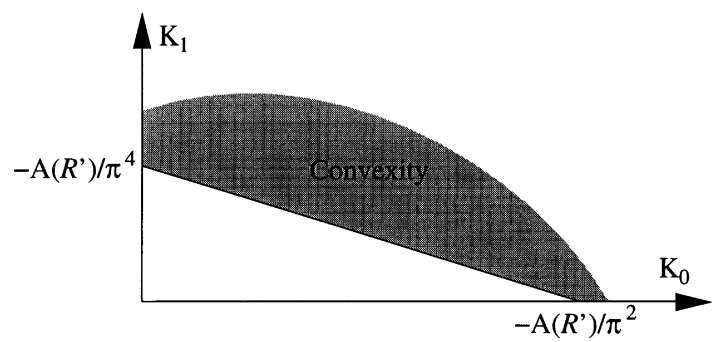

Fig. 1. Shaded area is the region in the $K_{0}-K_{1}$ plane that yields a convex problem in a region $\mathcal{R}^{\prime} \subseteq \mathcal{R}$.

$K_{0}$ and $K_{1}$ and on the potential $P(\mathbf{x})$. The potential, in turn, depends on the underlying image. To know how to choose $K_{0}$ and $K_{1}$ to maintain convexity, it is possible, in principle, to calculate $A\left(\mathcal{R}^{\prime}\right)$ for a given image or region within an image. In this and the following sections, however, we seek a more general understanding of the relationships between convexity of $\tilde{\varepsilon}(\mathbf{d})$ and object shape through a series of approximations.

In order to simplify our analysis, we first observe that as $N \rightarrow \infty$, Eq. (23) becomes independent of $N$. In particular, using a Taylor series expansion it is readily shown that

$\lim _{N \rightarrow \infty} 2 N^{2}(1-\cos (\pi / N))=\pi^{2}$.

This sequence converges fairly fast, so that for any practical value for $N$ (e.g. $N>30$ ), it is a very good approximation to use the limit in place of the expression. Using this approximation, Eq. (23) becomes

$A\left(\mathcal{R}^{\prime}\right)+K_{0} \pi^{2}+K_{1} \pi^{4}>0$.

As shown in Fig. 1, this condition gives a region in $K_{0}-K_{1}$ space which guarantees convexity of $\tilde{\varepsilon}(\mathbf{d})$.

We now seek to find simplified expressions for $h_{1}(\cdot)$ and $h_{2}(\cdot)$, which determine $A\left(\mathcal{R}^{\prime}\right)$, by expressing these functions in a local coordinate system. In particular, consider the curve of constant potential, the isopotential curve, passing through a point $\mathbf{x} \in \mathcal{R}$, and assume (at first) that its curvature at $\mathbf{x}$ is nonzero. We can then define a local polar coordinate system whose origin coincides with the center of curvature of this curve [28], as shown in Fig. 2. We now view the potential as a function of $r$ and $\phi$, the radius and angle in the local coordinate system, and seek expressions for $P_{x x}, P_{x y}$, and $P_{y y}$ at $\mathbf{x}$.

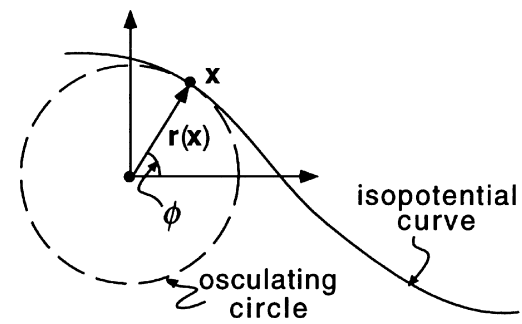

Fig. 2. Polar coordinate system centered at the center of curvature of the isopotential curve passing through $\mathbf{x}$. 
First we note that because of the way we defined the local coordinate system it can be shown that [20]

$P_{\phi}=P_{\phi \phi}=0$.

Using this fact, the chain rule yields

$P_{x}=P_{r} \cos \phi$

$P_{y}=P_{r} \sin \phi$

$P_{x x}=P_{r r} \cos ^{2} \phi+\frac{P_{r}}{r} \sin ^{2} \phi-2 \frac{P_{r \phi}}{r} \sin \phi \cos \phi$,

$P_{x y}=\left(P_{r r}-\frac{P_{r}}{r}\right) \cos \phi \sin \phi+\frac{P_{r \phi}}{r}\left(\cos ^{2} \phi-\sin ^{2} \phi\right)$,

$P_{y y}=P_{r r} \sin ^{2} \phi+\frac{P_{r}}{r} \cos ^{2} \phi+2 \frac{P_{r \phi}}{r} \sin \phi \cos \phi$.

where all functions are assumed to be evaluated at $\mathbf{x}$. Then we can rewrite $h_{1}$ and $h_{2}$ as

$$
\begin{aligned}
h_{1}(\mathbf{x})= & \frac{1}{4}\left(P_{r r}(\mathbf{x})+\frac{P_{r}(\mathbf{x})}{r(\mathbf{x})}\right. \\
& +\sqrt{\left.\left(P_{r r}(\mathbf{x})-\frac{P_{r}(\mathbf{x})}{r(\mathbf{x})}\right)^{2}+\frac{4 P_{r \phi}^{2}(\mathbf{x})}{r^{2}(\mathbf{x})}\right)}, \\
h_{2}(\mathbf{x})= & \frac{1}{4}\left(P_{r r}(\mathbf{x})+\frac{P_{r}(\mathbf{x})}{r(\mathbf{x})}\right. \\
& \left.-\sqrt{\left(P_{r r}(\mathbf{x})-\frac{P_{r}(\mathbf{x})}{r(\mathbf{x})}\right)^{2}+\frac{4 P_{r \phi}^{2}(\mathbf{x})}{r^{2}(\mathbf{x})}}\right),
\end{aligned}
$$

where $r(\mathbf{x})$ is the radius of curvature of the isopotential curve at $\mathbf{x}$ (see Fig. 2). Note that if we let $r \rightarrow \infty$ we obtain expressions for $h_{1}$ and $h_{2}$ for the case where the isopotential curve has zero curvature.

Now we are in position for our second approximation. We note that potentials used for locating boundaries have isopotential curves that are nearly parallel to each other, and that are nearly parallel to the boundary ${ }^{1}$. This is especially true when the boundaries are smooth or the point $\mathbf{x}$ is very near the desired boundary. In this case the term $P_{r \phi}(\mathbf{x})$ in Eqs. (28a) and (28b) is negligible and we can assume that $P_{r \phi}(\mathbf{x}) \approx 0$.

Introducing this approximation to Eqs. (28a) and (28b) we obtain

$$
\begin{aligned}
& h_{1}(\mathbf{x}) \approx \frac{1}{4}\left(P_{r r}(\mathbf{x})+\frac{P_{r}(\mathbf{x})}{r(\mathbf{x})}+\left|\left(P_{r r}(\mathbf{x})-\frac{P_{r}(\mathbf{x})}{r(\mathbf{x})}\right)\right|\right), \\
& h_{2}(\mathbf{x}) \approx \frac{1}{4}\left(P_{r r}(\mathbf{x})+\frac{P_{r}(\mathbf{x})}{r(\mathbf{x})}-\left|\left(P_{r r}(\mathbf{x})-\frac{P_{r}(\mathbf{x})}{r(\mathbf{x})}\right)\right|\right)
\end{aligned}
$$

\footnotetext{
${ }^{1}$ The value of the potential along each isopotential curve increases with the distance from the underlying boundary.
}

By defining

$e_{1}(\mathbf{x})=\frac{1}{2} P_{r r}(\mathbf{x})$

$e_{2}(\mathbf{x})=\frac{1}{2} P_{r}(\mathbf{x}) / r(\mathbf{x})$,

it is straightforward to show that

$\min _{\mathbf{x} \in \mathcal{R}^{\prime}}\left\{h_{1}(\mathbf{x}), h_{2}(\mathbf{x})\right\}=\min _{\mathbf{x} \in \mathcal{R}^{\prime}}\left\{e_{1}(\mathbf{x}), e_{2}(\mathbf{x})\right\}$.

Therefore, to good approximation $A\left(\mathcal{R}^{\prime}\right)$ is given by

$A\left(\mathcal{R}^{\prime}\right) \approx \min _{\mathbf{x} \in \mathcal{R}^{\prime}}\left\{e_{1}(\mathbf{x}), e_{2}(\mathbf{x})\right\}$.

We have now arrived to the main conclusion of this section: under the approximations described above, the analysis of the convexity of VP reduces to the study of the functions $e_{1}(\cdot)$ and $e_{2}(\cdot)$. We use this result in the following section, in which we study the convexity of four particular active contour potentials, and we show that under certain assumptions $e_{1}(\cdot)$ and $e_{2}(\cdot)$ are functions of only one variable, the radius of curvature $r$ of the isopotential curve at $\mathbf{x}$, which greatly simplifies the convexity analysis.

\section{Specific examples of the potential}

In this section we apply the convexity analysis of Section 3 to four particular active contour potentials, which are designed to find and parameterize boundaries. We derive explicit expressions for $A(\cdot)$ in Eq. (30) for all four potentials and, based on these expressions, we analyze the condition in (convexity). This analysis reveals important correlations between the uniqueness of the solution of VP, the selection of its regularization parameters, the initialization of the active contour relative to the boundary, and the geometric characteristics of the boundary.

\subsection{Ribbons}

In order to analyze all four potentials in a unified framework, we use an idealized model for object boundaries: a ribbon of thickness $w$ (see Fig. 3). Convolutional and morphological edge detection operations often produce edge maps resembling thin ribbons, whose thickness is directly related to the size of the kernel of the edge-detection

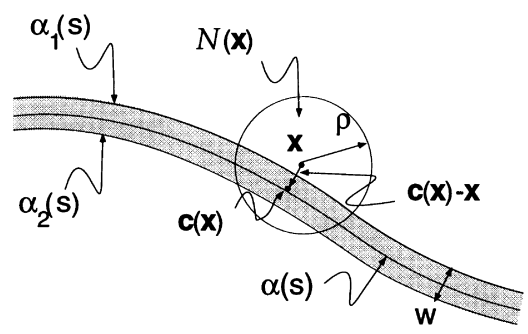

Fig. 3. Geometry of a ribbon with spine $\boldsymbol{\alpha}(s)$ and $\boldsymbol{\alpha}_{2}(s)$. 
operator. We denote by $C$ the collection of points belonging to a ribbon, and we define the mass of the ribbon to be

$m(\mathbf{x})=\left\{\begin{array}{cc}1 / w, & \mathbf{x} \in C, \\ 0, & \text { otherwise, }\end{array}\right.$

where the constant $1 / w$ is a normalization factor that makes the total mass of the ribbon equal to its length $L$. We note that as $w$ approaches zero, $m$ approaches a Dirac function at $C$, which defines an ideal boundary. We also denote, $\boldsymbol{\alpha}(s)$, $s \in[0,1]$, a parametrization of the spine of a ribbon; this is the desired solution to VP. Finally, we denote $\boldsymbol{\alpha}_{1}(s)$ and $\boldsymbol{\alpha}_{2}(s)$ the two outer curves defining the ribbon (Fig. 3).

\subsection{Ribbon potentials}

In order to use the variational framework of VP to find $\boldsymbol{\alpha}(s)$, we consider four different potentials, which are all based on the geometry of ribbons and are designed to take their minimum value in the vicinity of the spine of a ribbon. The first two, denoted $P_{1}$ and $P_{2}$, were introduced in Refs. $[24,13]$ in the context of cortical mapping, and were subsequently used [14] for estimating the central axis of tube-like objects. The last two, denoted $P_{3}$ and $P_{4}$, are variants of the potentials commonly used in snake models $[1,23]$. In order to define $P_{1}$ and $P_{2}$, we first introduce the center of mass function $\mathbf{c}(\mathbf{x})$, which is the center of the mass of the ribbon included in a circular neighborhood $\mathcal{N}(\mathbf{x})$ centered at $\mathbf{x}$ (see Fig. 3). We notice that, for sufficiently small neighborhood radius $\rho$, for any point $\mathbf{x}$ on the spine of the ribbon,

$\mathbf{c}(\mathbf{x}) \approx \mathbf{x}$.

Accordingly, we define $P_{1}$ and $P_{2}$ to be minimal on points which coincide with the center of the mass of the ribbon present in their neighborhood:

$P_{1}(\mathbf{x})=\|\left(\mathbf{x}-\mathbf{c}(\mathbf{x}) \|^{2}\right.$,

$P_{2}(\mathbf{x})=2 \int_{l=0}^{1}\|\mathbf{y}(l)-\mathbf{c}(\mathbf{y}(l))\| d l$,

where $\mathbf{y}(l), l \in[0,1]$, is a parameterization of the line of flow $^{2}$ connecting an image point $\mathbf{x}=\mathbf{y}(0)$ with the the minimum potential curve. The minimum potential curve for $P_{1}$ and $P_{2}$ is the collection of points satisfying the following condition:

$\mathbf{x}=\mathbf{c}(\mathbf{x})$.

We note that if the diameter of the disk $\mathcal{N}(\cdot)$ is much larger than the width of the ribbon, then Eq. (32) is not necessarily satisfied on the spine of a ribbon. Therefore, in the implementation of an active contour algorithm using $P_{1}$ or $P_{2}$, the neighborhood size should gradually decrease as the active contour approaches the spine [20]. For the purposes of the

\footnotetext{
${ }^{2}$ The line of flow is the trajectory of a particle under the influence of a potential gradient.
}

convexity analysis, we will assume that $\rho$ is sufficiently small so that Eq. (32) is satisfied on the spine.

From Eqs. (33) and (34) we easily see that the potentials $P_{1}$ and $P_{2}$ are minimal and equal to zero close to the spine of the ribbon, and precisely on the points satisfying Eq. (35), and gradually increase with increasing distance from it. Accordingly, the external force field induced by both $P_{1}$ or $P_{2}$ is a restoring force field tending to deform an active contour toward the spine.

In addition to $P_{1}$ and $P_{2}$, we also consider the following two potentials:

$P_{3}(\mathbf{x})=-\|m(\mathbf{x}) * g(\mathbf{x})\|$,

$P_{4}(\mathbf{x})=-\|m(\mathbf{x}) * g(\mathbf{x})\|^{2}$.

Here, $g(\mathbf{x})$ is defined as follows:

$g(\mathbf{x})= \begin{cases}1, & \mathbf{x} \in \mathcal{N}(\mathbf{0}), \\ 0, & \text { elsewhere, }\end{cases}$

where $\mathcal{N}(\mathbf{x})$ denotes a circular neighborhood centered on $\mathbf{x}$. These potentials are similar to the usual snake potentials $[1,23]$, but differ in the following respects:

1. $P_{3}$ and $P_{4}$ use the binary mass function $m(\cdot)$ instead of the magnitude of the image intensity gradient which is customary in snake models. A binary mass can be readily obtained by thresholding the edge map obtained through the image gradient.

2. $P_{3}$ and $P_{4}$ use the "disk" function of Eq. (37), instead of a Gaussian smoothing function. These modifications are introduced here in order to simplify the convexity analysis and allow direct comparisons between all four models.

We will assume throughout our development that $P_{1}, P_{2}$, $P_{3}$, and $P_{4}$ are defined at $\mathbf{x}$, if the disk $\mathcal{N}(\mathbf{x})$ intersects both $\boldsymbol{\alpha}_{1}(s)$ and $\boldsymbol{\alpha}_{2}(s)$ (see Fig. 3). Accordingly, the domain of definition of these potentials is

$$
\begin{gathered}
\mathcal{R}=\left\{\mathbf{x} \in \mathbb{R}^{2}:\left\{\mathcal{N}(\mathbf{x}) \cap \boldsymbol{\alpha}_{1}(s) \neq \varnothing\right\}\right. \\
\text { and } \left.\left\{\mathcal{N}(\mathbf{x}) \cap \boldsymbol{\alpha}_{2}(s) \neq \varnothing\right\}\right\} .
\end{gathered}
$$

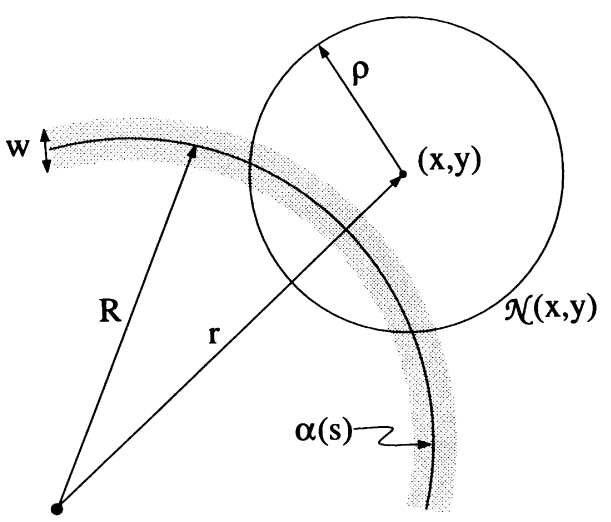

Fig. 4. Intersection of a disk $\mathcal{N}(x, y)$ around a point $(x, y)$ with a thin ribbon of width $w$. 
We note here that outside $\mathcal{R}$, the energy function $\varepsilon$ is equal to $\varepsilon_{\mathrm{I}}$, and therefore the active contour contracts under the influence of its internal elastic forces, until it reaches the vicinity of the ribbon. Since in that region $\varepsilon$ is obviously convex, we will restrict our convexity analysis to $R$.

\subsection{Convexity of ribbon models}

Having defined the four potentials, we now turn our attention to $A(\cdot)$ and the convexity condition in (25). In order to obtain explicit expressions for $A(\cdot)$, we need to make certain assumptions about the shape of the underlying boundary. In particular, we approximate the intersections of the ribbon

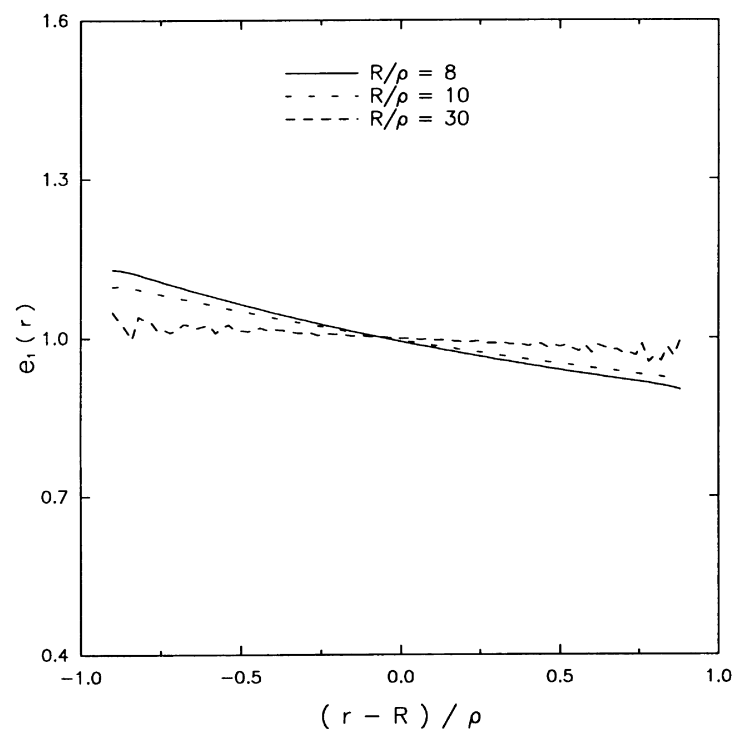

(a)

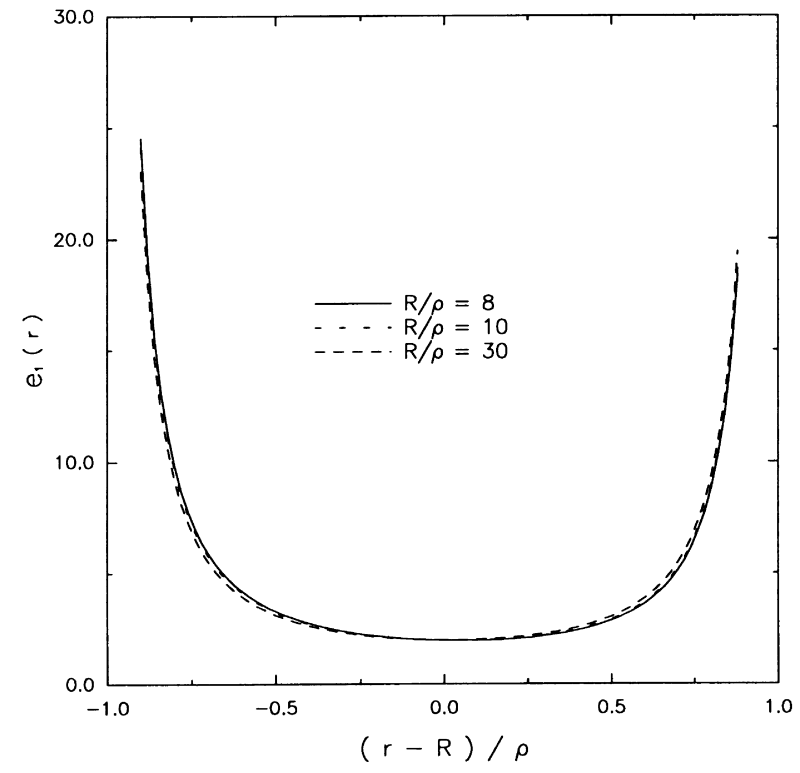

(c) with neighborhoods of active contour points by arcs of radius $R$ and thickness $w$, as shown in Fig. 4. Under this approximation, $P_{r \phi}$ is exactly zero, and $A(\cdot)$ is exactly given by Eq. (30). Moreover, $e_{1}(\cdot)$ and $e_{2}(\cdot)$ are functions only of the radial coordinate $r$. Therefore, the convexity analysis reduces to the study of the one-dimensional functions $e_{1}(r)$ and $e_{2}(r)$.

In Ref. [20], expressions for $e_{1}(r)$ and $e_{2}(r)$ were derived for all four potentials $P_{1}, P_{2}, P_{3}$, and $P_{4}$. These expressions depend on the curvature of the ribbon, the ribbon width, and on the neighborhood radius, which makes the display and interpretation of them a fairly difficult task. One way of displaying this information is shown in Figs 5 and 6, which plot $e_{1}(r)$ and $e_{2}(r)$ against $(r-R) / \rho$, for different

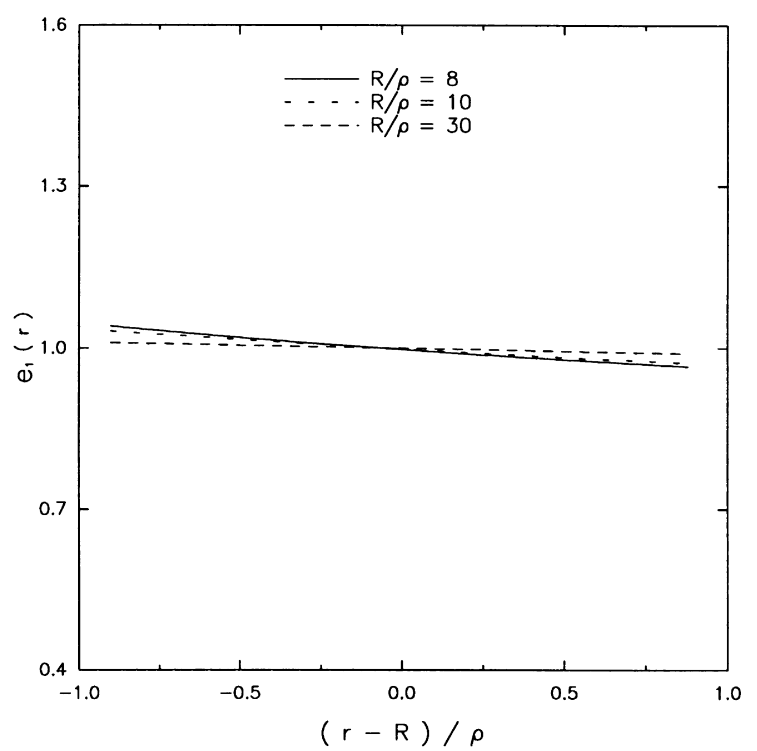

(b)

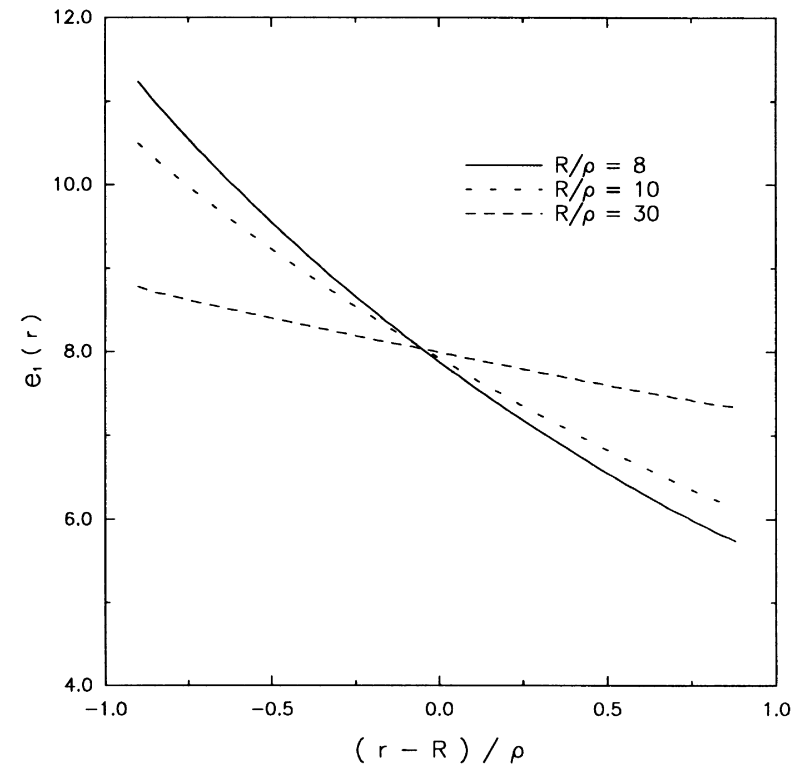

(d)

Fig. 5. Plots of $e_{1}(r)$ for different ratios $R / \rho$. (a) $P_{1}$, (b) $P_{2}$, (c) $P_{3}$, and (d) $P_{4}$. 
ratios $R / \rho$. Several conclusions can be drawn from these figures and the convexity condition in Eq. (25):

1. The function $e_{1}(r)$ (Fig. 5) is positive for $r \in[R+w / 2-$ $\rho, R-w / 2+\rho]$. Therefore, it is positive everywhere in the domain $R$ and its minimum value in $R$ is positive. Given Eqs. (25) and (30), this implies that $e_{1}(r)$ imposes no restriction on selecting a pair $\left(K_{0}, K_{1}\right)$ which yields a convex function.

2. The function $e_{2}(r)$ is always positive for $r \geq R$ (positive horizontal axis in Fig. 6). Therefore, both $e_{1}(r)$ and $e_{2}(r)$ are non-negative at all points in $\mathcal{R}$ for which

$r(\mathbf{x}) \geq R(\mathbf{x})$,

where $R(\mathbf{x})$ is the radius of curvature of the spine in $\mathcal{N}(\mathbf{x})$,

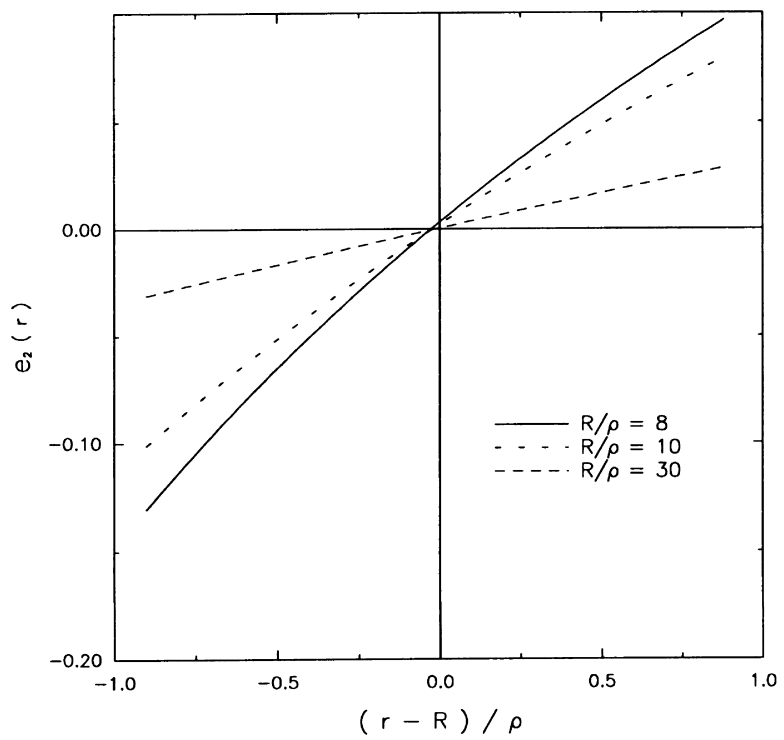

(a)

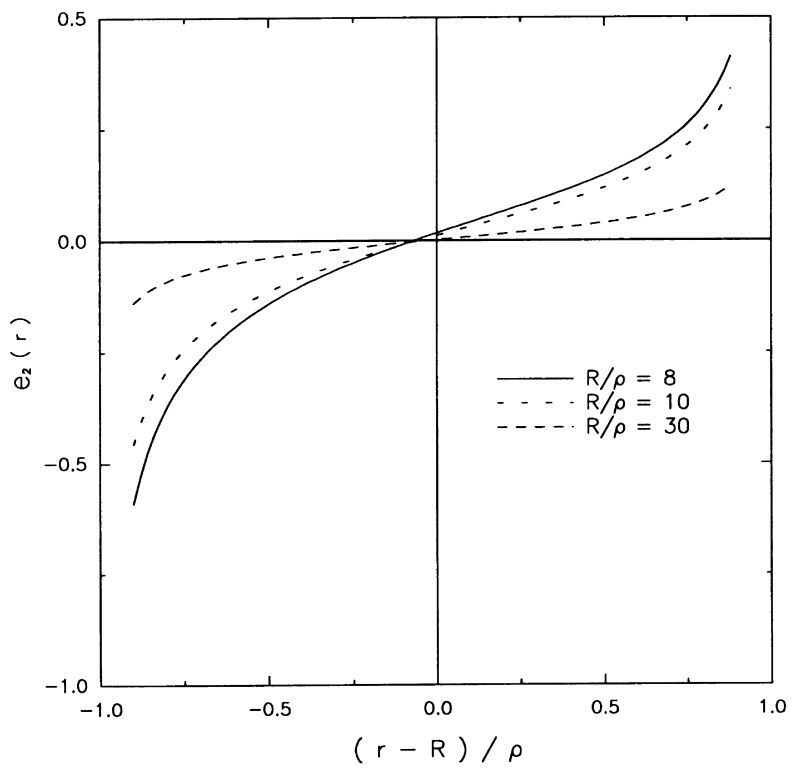

(c) and $r(\mathbf{x})$ is the radius of curvature of the isopotential curve passing through $\mathbf{x}$. Let $\mathcal{R}^{+}$be the collection of points satisfying Eq. (39), which can be shown to be a connected subdomain of $\mathcal{R}$ [20]. Then the convexity condition in Eq. (25) is satisfied everywhere in $\mathcal{R}^{+}$for all $\left(K_{0}, K_{1}\right)$ such that $K_{0}>0, K_{1}>0$. This region of convexity is shown schematically in Fig. 7. The implications of this result are important. In particular, an optimization method seeking a solution to the Euler equations of VP within the subdomain of convexity $\mathcal{R}^{+}$is guaranteed to converge to the global minimum in $\mathcal{R}^{+}$. A similar result was used [20] as the basis for a domain adaptive active contour algorithm which restricted the active contour in $\mathcal{R}^{+}$; therefore, avoiding local minima.

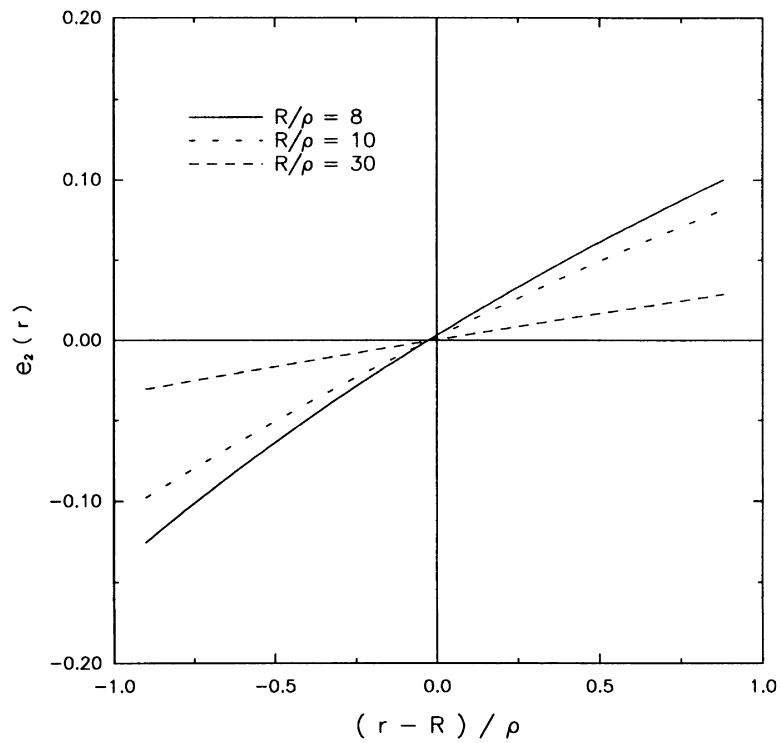

(b)

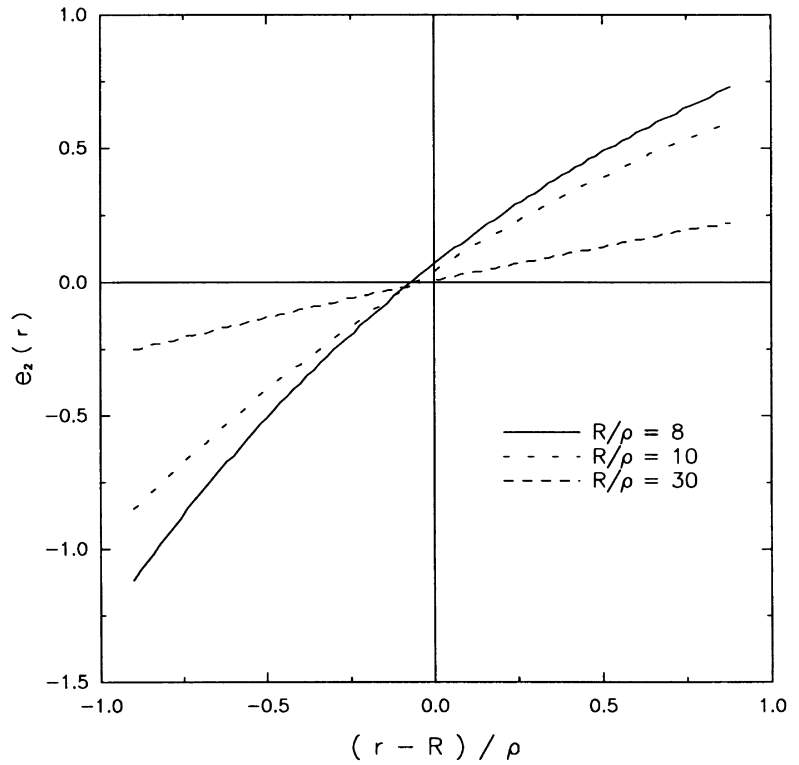

(d)

Fig. 6. Plots of $e_{2}(r)$ for different ratios $R / \rho$. (a) $P_{1}$, (b) $P_{2}$, (c) $P_{3}$, and (d) $P_{4}$. 


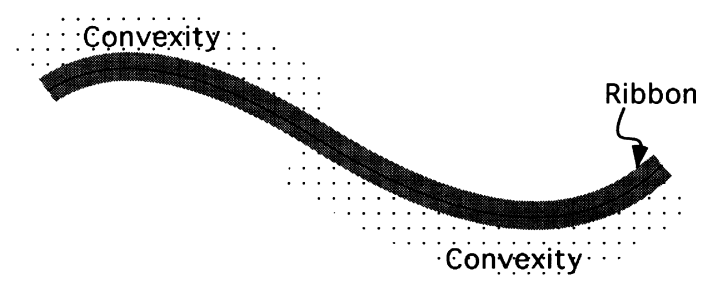

Fig. 7. Region of convexity of VP. This region includes the collection of points for which $r(\mathbf{x}) \geq R(\mathbf{x})$ (see text), or equivalently the points which face the convex part of the folds of the ribbon.

3. For $r<R$ (negative horizontal axis in Fig. 6), $e_{2}$ is negative for all potentials, and it is considerably smaller for $P_{3}$ and $P_{4}$ than for $P_{1}$ and $P_{2}$. Therefore, the minimum value of $e_{2}$ in $\mathcal{R}$ for the $P_{3}$ and $P_{4}$ formulations is smaller than that of the formulations using $P_{1}$ and $P_{2}$. Considering Eqs. (25) and (30) this implies that $K_{0}$ and $K_{1}$ should be larger for the models using $P_{3}$ and $P_{4}$. Consequently, the elasticity forces in the $P_{1}$ and $P_{2}$ formulations can be smaller while still maintaining the convexity of VP. This is important since large elasticity forces often cause oversmoothing of boundaries [13].

4. The plots of Fig. 6 show that $e_{2}(r)$ decreases in absolute value with the distance from the spine. This implies that as the active contour gets closer to the spine, smaller values of $K_{0}$ and $K_{1}$ can be used. Similarly, if an active contour is initialized close to the spine, much smaller $K_{0}$ and $K_{1}$ are required to maintain convexity. A similar result motivated the development of a time-adaptive active contour algorithm [24], which was shown to improve the ability of the active contour to bypass local minima considerably, by gradually reducing the elasticity parameters.

\section{Summary}

This paper studied a general active contour formulation (VP), and derived conditions for the convexity of its energy function. The derived condition quantitatively expressed the relation between the convexity of the energy function and the selection of the regularization parameters. The results were then applied to four special cases of VP designed to find parametrizations of boundaries, which are modeled as ribbons.

The first main conclusion drawn from this analysis is that in order for the energy function of VP to be convex, $K_{0}$ and $K_{1}$ should be selected from a specific domain in the $K_{0}-K_{1}$ plane. This region is determined by the potential $P$ of the active contour formulation. The application of this result to four particular potentials in Section 4 lead to the second conclusion of our analysis. Specifically, it was shown that, under certain assumptions, there exists a connected domain where the energy function is convex for any pair of positive regularization constants, for all four potentials for boundary parameterization. This implies that an active contour algorithm can avoid local minima by searching for a solution within the domain of convexity. The development of such domain adaptive active contour algorithms [20] is a direction of further research originating from the convexity analysis presented in this paper.

\section{Acknowledgements}

The authors would like thank Nick Bryan for his support to this work. This research was partly supported by the NSF grant MIP-9350336 and the NIH grant NICHD P50HD 25806-03. A condensed version of this paper appeared in the Proceedings of the IEEE Conference on Computer Vision and Pattern Recognition, June 1996.

\section{References}

[1] M. Kass, A. Witkin, D. Terzopoulos, Snakes: active contour models, International Journal of Computer Vision 1 (1988) 321-331.

[2] S. Kichenassamy, A. Kumar, P. Olver, A. Tannenbaum, A. Yezzi, Gradient flows and geometric active contours. Proc. Int. Conf. on Computer Vision, ICCV'95, pp. 810-815, 1995.

[3] R. Malladi, J.A. Sethian, B.C. Vemuri, Shape modeling with front propagation, IEEE Trans. on Pattern Analysis and Machine Intelligence 17 (1995) 158-177.

[4] V. Caselles, F. Catte, T. Coll, F. Dibos, A geometric model for active contours in image processing, Technical Report 9210, CEREMADE, Universite Paris Dauphine, 1992.

[5] F. Leymarie, M. Levine, New method for shape description based on an active contour model, SPIE Proc., Intelligent Robots and Computer Vision VIII: Algorithms and Techniques 1192 (1989) 536-547.

[6] F. Leymarie, M.D. Levine, Tracking deformable objects in the plane using an active contour model, IEEE Trans. on Pattern Analysis and Machine Intelligence 15 (6) (1993) 617-634.

[7] D.J. Williams, M. Shah, A fast algorithm for active contours and curvature estimation, CVGIP: Image Understanding 55 (1992) $14-26$.

[8] A.A. Amini, S.S. Tehrani, T.E. Weymouth, Using dynamic programming for minimizing the energy of active contours in the presence of hard constraints. Proc. Int. Conf. on Computer Vision, pp. 95-99, 1988.

[9] M.E. Hyche, N.F. Ezquerra, R. Mullick, Spatiotemporal detection of arterial structure using active contours, SPIE Proc. Visualization in Biomedical Computing 1808 (1992) 52-62.

[10] R. Samadani, D. Mihovilovic, C.R. Clauer, G. Wiederhold, J.D. Craven, L.A. Frank, Evaluation of an elastic curve technique for finding the auroral oval from satellite images automatically, IEEE Trans. on Geoscience and Remote Sensing 28 (4) (1990) 590-597.

[11] R. Samadani, Changes in connectivity in active contour models. Proc. Workshop on Visual Motion, pp. 337-343, 1989.

[12] K.F. Lai, Deformable contours: modeling, extraction, detection, and classification. PhD thesis, University of Wisconsin, Madison, 1994.

[13] C.A. Davatzikos, J.L. Prince, An active contour model for mapping the cortex, IEEE Trans. on Medical Imaging 14 (1995) 65-80.

[14] W. Lawton, Mathematical methods for active geometry. Annals of Numerical Mathematics, eds. C. Micchelli, H. Said, (CAGD conference, Penang, 1994), 1, 1995.

[15] L. Liu, G. Chunck, C.R. Meyer, Optimal contour approximation by deformable piecewise cubic splines. Proc. IEEE Conf. on Computer Vision and Pattern Recognition, pp. 638-643, 1990. 
[16] R. Cedeberg, Chain-link coding and segmentation for raster scan devices, Computer Graphics and Image Processing 10 (1979) 224-234.

[17] A.K. Jain, Fundamentals of Digital Image Processing. Prentice Hall, 1989.

[18] F. Leymarie, Tracking and describing deformable objects using active contour models. Technical Report TR-CIM-90-9, McGill University, 1990.

[19] M.O. Berger, R. Mohr, Towards autonomy in active contour models Proc. 10th Int. Conf. on Pattern Recognition, pp. 847-851, 1990.

[20] C. Davatzikos, Model-based boundary mapping with applications to medical imaging. PhD thesis, Johns Hopkins University, 1994.

[21] D. Marr, E.C. Hildreth, Theory of edge detection, Proceedings of the Royal Society of London B270 (1980) 187-217.

[22] J.F. Rivest, P. Soille, S. Beucher, Morphological gradients, Journal of Electronic Imaging 2 (4) (1993) 326-336.
[23] L.D. Cohen, I. Cohen, Finite-element methods for active contour models and balloons for 2D and 3D images, IEEE Trans. Pattern Analysis and Machine Intelligence 15 (11) (1993) 1131-1147.

[24] G.A. Davatzikos, J.L. Prince, Adaptive active contour algorithms for extracting and mapping thick curves. Proc. IEEE Conf. on Computer Vision and Pattern Recognition, pp. 524-529, 1993.

[25] F.B. Hildebrand, Advanced Calculus for Applications. Prentice-Hall, Englewood Cliffs, NJ, 1976.

[26] U. Grenander, G. Szego, Toeplitz Forms and their Applications, University of California Press, 1958.

[27] L.D. Cohen, A. Gorre, On the convexity of the active contour energy. Proc. of GRETSI, September 1995.

[28] R. Millman, G. Parker, Elements of Differential Geometry. Prentice Hall, 1977. 